\title{
Structure and function relationship of Zebrafish embryonic heart from confocal microscopy images
}

\author{
Abbas N. Moghaddam ${ }^{\mathrm{a}}$, Arian Forouhar ${ }^{\mathrm{a}}$, Michael Liebling ${ }^{\mathrm{a}}$, Huai-Jen Tsai ${ }^{\mathrm{b}}$ and Morteza Gharib ${ }^{\mathrm{a}}$ \\ ${ }^{a}$ California Institute of Technology, Pasadena, CA, USA 91125-0001; \\ ${ }^{\mathrm{b}}$ National Taiwan University, Taiwan
}

\begin{abstract}
Confocal microscopy enables us to track myocytes in the embryonic zebrafish heart. The Zeiss LSM 5 Live high speed confocal microscope has been used to take optical sections (at $3 \mu \mathrm{m}$ intervals and 151 frames per second) through a fluorescently labeled zebrafish heart at two developmental stages (26 and 34 hours post fertilization (hpf)). This data provides unique information allowing us to conjecture on the morphology and biomechanics of the developing vertebrate heart. Nevertheless, the myocytes, whose positions could be determined in a reliable manner, were located sparsely and mostly in one side of the heart tube. This difficulty was overcome using computational methods, that give longitudinal, radial and circumferential displacements of the myocytes as well as their contractile behavior. Applied strain analysis has shown that in the early embryonic heart tube, only the caudal region (near the in-flow) and another point in the middle of the tube can be active; the rest appears to be mostly passive. This statement is based on the delay between major strain and displacement which a material point experiences. Wave-like propagation of all three components of the displacement, especially in the circumferential direction, as well as the almost-periodic changes of the maximum strain support the hypothesis of helical muscle structure embedded in the tube. Changes of geometry in the embryonic heart after several hours are used to verify speculations about the structure based on the earlier images and aforementioned methods.
\end{abstract}

Keywords: cardiac mechanics, confocal microscopy, Embryonic heart, image-based biomechanical models, strain, structure, function.

\section{INTRODUCTION}

Heart disease is the most common and costly medical problem in the modern world, which makes understanding of the heart function a priority in medical research. The limited knowledge in how the form and function of the heart are related continues to restrict the ability to design strategies for effective treatments of heart failure [1]. The embryonic heart is unique in that it starts functioning in the early stages of its growth and never stops functioning during structural changes; therefore, the structure of the embryonic heart can be investigated always through its function. It can in turn help in understanding the form-function relation in the grown heart which is still under debate [2].

The action of the heart tube as a unidirectional pump suggests that it is a peristaltic pump [3,4], however, some other observations show that it cannot be purely explained as a peristaltic pump [5]. The nonlinear relation of the flow and frequency of beating [5] as well as the large strain of the myocytes, way beyond the normal limit of the strain in the muscles, are two examples of these observations.

The way we may connect the form and function is to track the tissues. High speed laser confocal microscopy is an exceptional tool for tracking the genetically labeled mycytes. However, light scattering decreases the effective depth at which we may get clear images and therefore acquired data only consists of a sparse arrangement of the material points mainly from one side of the heart tube. In this study we have focused on the computational tools that we may utilize to extract reliable information out of these images which provides quantitative evidences for speculations about the structure of the embryonic heart.

\section{METHODOLOGY}

\subsection{Experimental methods}

The zebrafish (Danio rerio) has been used as our animal model in the study on the early heart tube dynamics. This model has shown great performance for cardiogenetic studies as its transparent embryos in the eggs provide optical access to the earliest stages of heart development. For this experiment, transgenic fish with fluorescently labeled myocardia $\operatorname{Tg}(\mathrm{cmlc2}:: \mathrm{GFP})$ have been used [6]. A Zeiss LSM 5 Live high speed confocal microscope has been used to take optical

Medical Imaging 2006: Physiology, Function, and Structure from Medical Images, edited by Armando Manduca, Amir A. Amini, Proc. of SPIE Vol. 6143,

614301, (2006) - 1605-7422/06/\$15 - doi: 10.1117/12.652779

Proc. of SPIE Vol. $6143614301-1$ 
sections at $3 \mu \mathrm{m}$ intervals and 151 frames per second. A total of 120 images were acquired which cover two complete periods of the cardiac cycle. Imaging was performed on 26 and 34 hours post fertilization (hpf), when the heart is still a valveless tube. These sections (about 40) were synchronized using a wavelet-based registration algorithm to give 4D (3D+time) data [Error! Reference source not found.]. This data was then imported into the Imaris software to collect automated 3D position information for the cells. These images from the zebrafish embryonic heart eventually lead to conjecture about the formation process of the grown heart.

\subsection{Computational methods}

\section{1) Directions:}

Image processing and mathematical tools have been used next for calculation of the displacement waves and strain. To distinguish and measure the longitudinal, radial and circumferential displacement, first these directions should be defined at every given material point over the wall of this twisted and declined tube. For each given point, to calculate the normal vector to the tube surface, $\hat{n}$, we consider a small neighborhood of that point and collect the coordinates of all material points located inside that neighborhood in a matrix as shown in equation (1). Then the covariance matrix of the coordinate matrix is calculated:

$$
\begin{gathered}
C=\left[\begin{array}{lll}
x_{i} & y_{i} & z_{i}
\end{array}\right]_{n \times 3} \\
A=\operatorname{cov}(C)
\end{gathered}
$$

The eigenvector corresponding to the smallest eigenvalue of the covariance matrix gives an acceptable estimate of the normal vector [8]. It simply shows the direction in which the positions of the points in that neighborhood have the minimum changes.

$$
\begin{gathered}
A \cdot V_{i}=\lambda_{i} \cdot V_{i} \\
\left|\lambda_{1}\right|<\left|\lambda_{2}\right|<\left|\lambda_{3}\right| \Rightarrow \hat{n} \approx \hat{V}_{1}
\end{gathered}
$$

To determine the two other directions we had to extend this method. For longitudinal direction, we first consider the rough overall direction of the tube by choosing one point at each of its ends. Then we determine a non isotropic neighborhood for each point using an ellipsoid whose main axis is parallel to that direction and centered in the aforementioned point, as shown in Figure 2 . The covariance matrix of the coordinates of these neighboring points should be calculated next and its eigenvalues and eigenvectors determined. The eigenvector corresponding to the largest eigenvalue gives the direction in which the points are most distributed and so should be close to the

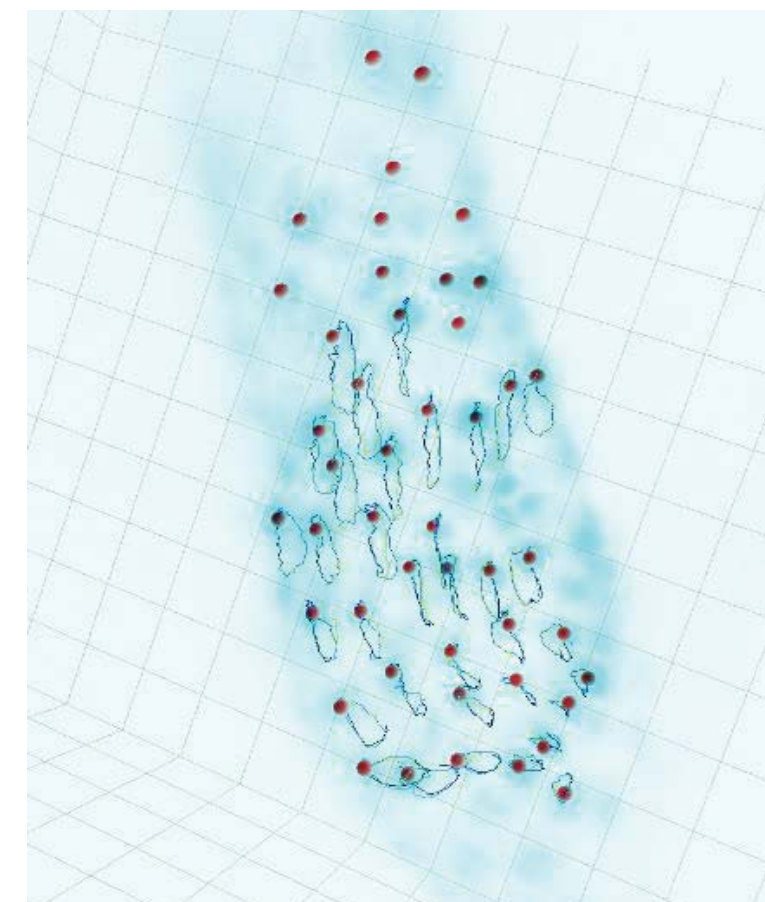

Figure 1. An image obtained with the high speed confocal microscope (Zeiss LSM 5 Live) with optical sections $(3 \mu \mathrm{m}$ intervals, $151 \mathrm{fps}$ ) through a 26 hour post fertilization zebrafish, Tg (cmlc2::GFP), embryo. These sections (about 40) were synchronized using a wavelet-based registration algorithm to give $4 \mathrm{D}(3 \mathrm{D}+$ time $)$ data. This data was imported into the Imaris software to collect automated 3D position information for the cells. For some of the points the trajectory they traverse has been shown.

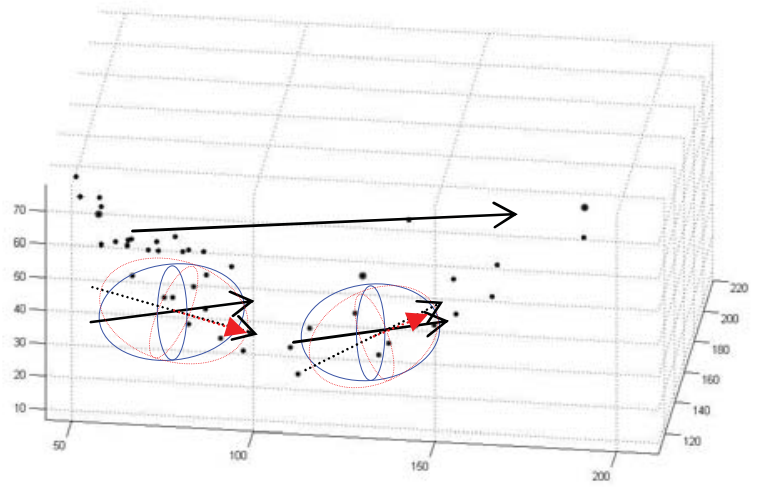

Figure 2. The procedure for finding the longitudinal unit vectors for two sample points is shown. In the beginning the blue ellipsoids are all in the same orientation, i.e. parallel to the overall direction of the tube (solid arrows.) Then after one trial a direction closer to the real longitudinal unit vectors is found (red, dashed arrows). The calculations are repeated for the new neighborhood determined by the ellipsoids oriented in the new direction. (red, dashed ellipsoids.) 
longitudinal unit vector, $\hat{t}$. To have a more accurate approximation, for each point, this procedure is repeated by substituting its particular calculated direction instead of the overall direction as the axis of the aforementioned ellipsoid. This can help to correct the previous longitudinal unit vector. Figure 2 shows this procedure for two myocytes at one time point. Once these two unit vectors are determined, the third one, $\hat{c}$, which shows the circumferential direction, can easily be calculated by the cross product of the first two vectors, $\hat{c}=\hat{t} \times \hat{n}$, to form an independent right angle coordinate system at each point. For each time step, displacement of each point is expressed by its three components in its own coordinate system. Results of this algorithm are shown in Figure 3 and discussed in the Results section.

2) Lagrangian framework:

To track the myocytes along the tube, we have used a one-dimensional Lagrangian framework for our calculations. When the tube has not been contracted yet (rest situation), we divide all the material points in groups by sectioning the whole volume through successive thin layers perpendicular to the main axis of the tube. Layers have equal thickness but groups include different number of data points. These groups remain constant during the whole period. Although the three unit vectors, and therefore the three components of the movement, are independently calculated for each data point, the overall movement for each section is determined from their average over the points in each group.

We also determine a curvature for each group by calculating the approximate intersection of the normal vectors of its material points. This has been done through a least-squares error method. It should be noted that this curvature relates only to that part of the slice where we have enough data points. If the tube is twisted or bent, the curvature can be quite different for two sides of the tube.

3) Spatio-Temporal smoothing

The spatio-temporal resolution of the images is in the same order of the movement of the imaged particles and so the digitization noise is quite considerable when we have only a few data points in each group. To stabilize the calculations over noisy sparse data we have used a smoothing

Gaussian filter in both time and space.

4) Strain calculation

Calculation of the strain greatly helps in understanding the structure of the embryonic heart as will be discussed in next section. In the Lagrangian coordinate system described above we may easily find the 1D Lagrangian strain for the embryonic heart in its main axis direction. It takes place by a simple differentiation of the longitudinal movement of the particles. As shown in Figure 3 (a), if the tube experiences a deformation in the longitudinal direction, we may calculate the linear strain of that as:

$$
\text { strain }=\frac{\Delta L}{L_{1}}=\frac{L_{1}-L_{2}}{L_{1}}=\frac{d l_{2}-d l_{1}}{L_{1}}
$$

Now if we plot the longitudinal displacement versus the primary position as shown in the Figure 3(b), the strain equals the slope of the corresponding segment of this plot $(\tan (\alpha))$ which is the derivative of the plot at points located on $L_{l}$. If we call the displacement function $f(L)$, we may write:

$$
\text { strain }=\frac{d l_{2}-d l_{1}}{L_{1}}=\tan (\alpha)=\frac{d f}{d L}
$$

As we choose to work in the Lagrangian coordinate system, this will be calculated easily. To decrease the effect of noise we have calculated this derivative based on a least-squares method over a 7 points neighborhood of each point.

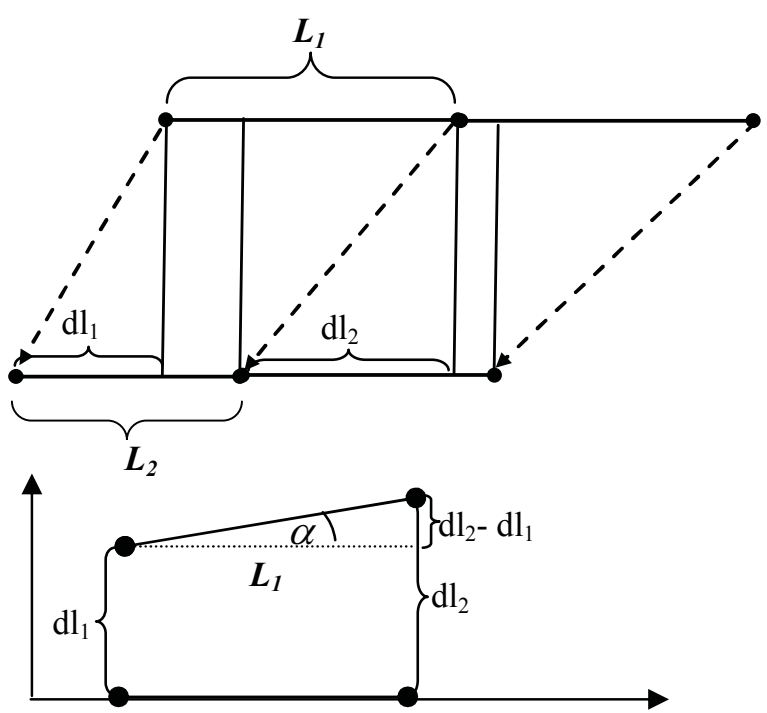

Figure3. (a) An example of $1 \mathrm{D}$ displacement. A segment of length $\mathrm{L}_{1}$ has been deformed to a segment of length $\mathrm{L}_{2}$. $\mathrm{dl}_{1}$ and $\mathrm{dl}_{2}$ are the longitudinal displacements of the two ends of this segment. (b) The plot of longitudinal displacement shows it as a function of primary length. 


\section{SYSTEM'S DYNAMICS}

Having an acceptable description of the three components of the displacement as well as the linear longitudinal strain through our computational methods, in this section we want to show how it may be related to the structure of the embryonic heart. Based on some other experiments, mentioned in the introduction, we may test the idea that only part of the tube moves actively and the rest moves only passively as an impedance pump [5]. The main idea is to consider the delay between contraction and displacement. Assuming that the tube is made from an active and a passive part, for the active part we expect to see its contraction (or strain) prior to or almost concurrent with its major displacement. However, for the passive part we expect to see the displacement first and then the shortening, mainly based on the propagation of the contraction wave generated by the active part in the tube.

In the longitudinal direction we first calculate the times at which considerable shortening and displacement happen and then measure the delay between them. Here we define the considerable shortening as the time at which the myocardial cells start experiencing a $10 \%$ or more shortening (negative) strain, provided it's not less than one third of the maximum strain. The time of displacement is also defined for each slice over the tube as the time of reaching to the $90 \%$ of maximum displacement for that slice.

Another factor that can help predict the active and passiveness of the segments can be derived from the circumferential movement (twist) in the tube. Regions in which the twist starts should be active parts of the tube. Especially if there is a close pair of twists in opposite directions, it suggests a spring like structure for the active element embedded in the tube wall.

There are still other potential factors that help us relate the functionality and structure of the embryonic heart. For example to discern the active parts we may search for the place of the maximum narrowing that decays as it travels to the other parts of the tube. Or we may calculate the pattern of the maximum strain that happens for each slice during the whole period of beating. In the next section we try to use these factors based on the two data sets that show 26 and 34 hpf movement of the heart.

\section{RESULTS}

\subsection{Calculation of the directions}

The methods described in Section 2 were used for both data sets. The results are shown in Figures 4 and 5 for 26 and 34 hpf, respectively, where in Figure 4 (a) and (b) the left sides show the beginning of the tube (in-flow) and the right sides show the end (out-flow). The accuracy of the method can be validated visually. Figure 4 shows that the 26 hpf data contains only some points from underneath the tube. For $34 \mathrm{hpf}$, data points are mainly located in the lower part of the tube but the data is incomplete in the very bottom of the heart. It caused one of the vectors to have rotated considerably from its correct direction.

Figures also show that the concentration of the captured points at the beginning of the tube is larger than at its end. It should be noted that not all the myocytes in the scanned region have reliable projections and therefore, a larger number of points in these graphs does not guarantee the presence of more myocytes.

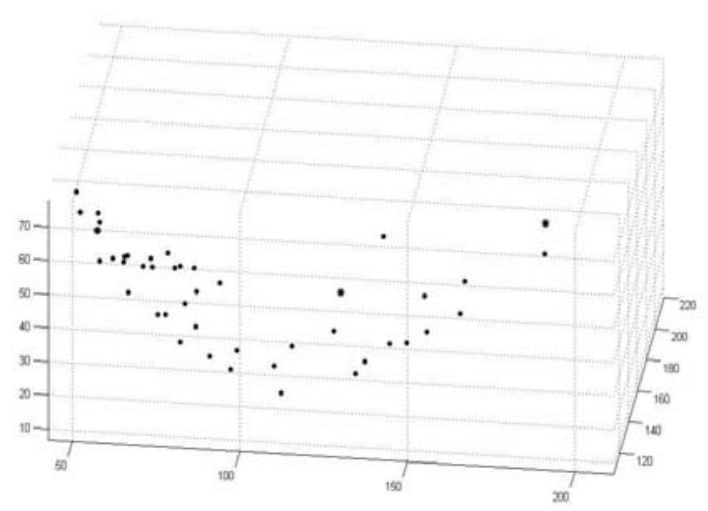

(a)

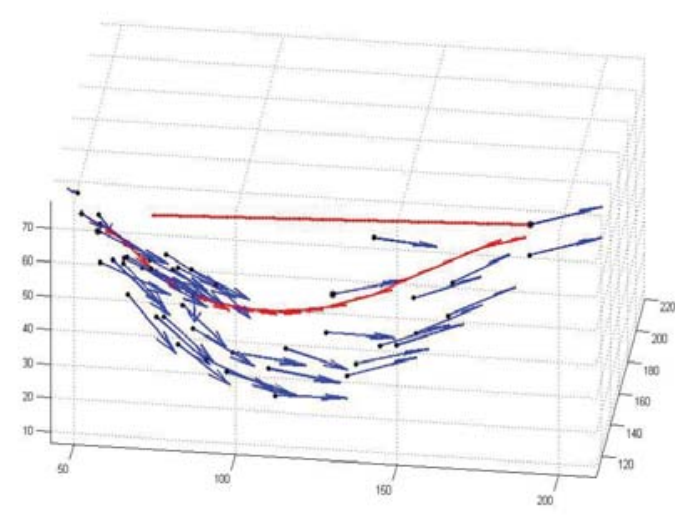

(b)

Figure 4. (a) The positions of myocytes in the $26 \mathrm{hpf}$ embryonic heart are shown. (b) Shows the same points along with the calculated longitudinal unit vectors which are blue. The straight red line shows the overall direction while the red curve shows a sample calculated path on the tube wall. 


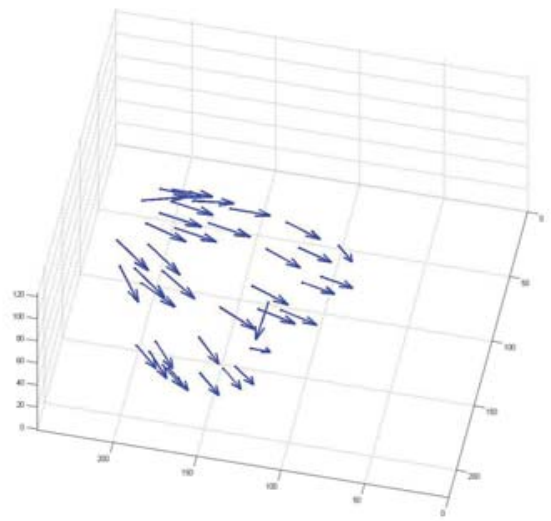

(a)

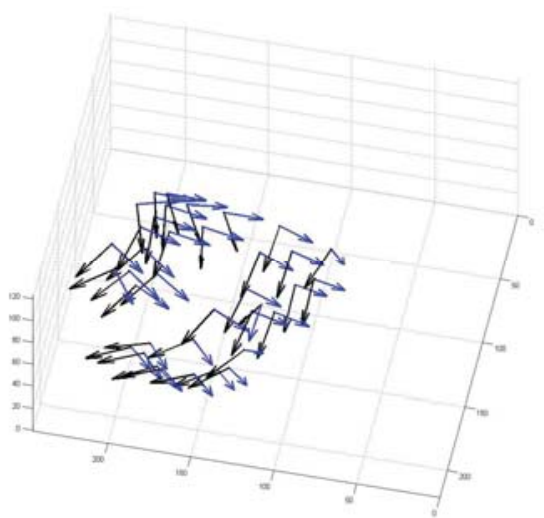

(b)

Figure 5. (a) Myocytes and the calculated longitudinal unit vectors for $34 \mathrm{hpf}$ embryonic heart. (b) Same as (a), but the circumferential unit vectors are added in black. These vectors give a better sense of the 3D configuration visualization of the whole tube.

\subsection{Slice Displacement}

The movements of thin layers perpendicular to the main axis of the tube have been plotted for $26 \mathrm{hpf}$ data set in Figure 6 .
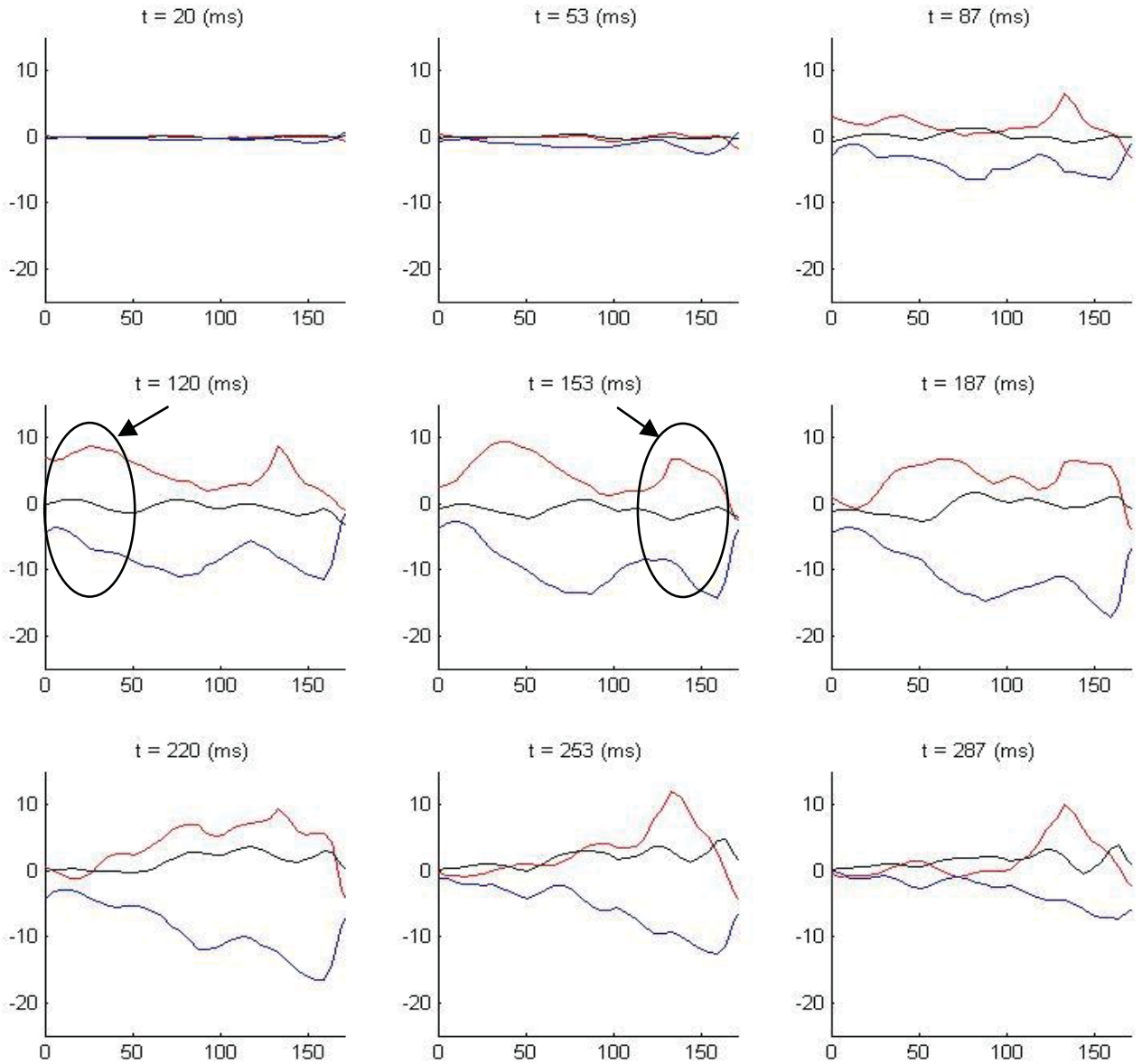

Figure 6. The longitudinal, radial and circumferential displacements for the $26 \mathrm{hpf}$ dataset are shown respectively in blue, red and black. All units are in $\mu \mathrm{m}$. 
Each plot shows all three components of the displacement for a specific time. The longitudinal, radial, and circumferential displacements are shown respectively in blue, red, and black. All units are in $\mu \mathrm{m}$. It shows that all three displacements have a wave-like motion from left to right, which is the direction of the flow.

Plots in Figure 6 show that there is a general movement in the beginning towards the beginning of the tube (negative values for the blue curve), however, only two regions show negative slopes, which corresponds to shortening. These two regions have been marked with ovals in two subplots. These two sites also contain the peak of the red plots, which show the maximum narrowing in the tube. The circumferential movements are not so significant at this stage. The timing analysis for testing active points was done on this dataset and the results are shown in Figure 7 below.

It shows that only a small beginning portion of the tube (about $20 \mu \mathrm{m}$ ) is active. However, the small delay near the position of $60 \mu \mathrm{m}$ may show some active behavior there. The plot shows another local minimum around $120 \mu \mathrm{m}$, which is the place of the second oval in Figure 6. The red dashed line represents the maximum derivative of the longitudinal displacement (shortening strain). It supports the existence of some activity near $60 \mu \mathrm{m}$. Its frequent alteration also may be related to some kind of spiral structure in the tube. It also may show different material properties at various locations.

To further investigate these conjectures, we have done the same procedure for the $34 \mathrm{hpf}$ dataset. Results are shown in Figure 8. This time, the two marked positions show their special behavior even more strongly, especially in the first one. It should be noted that the horizontal axis in Figures 6 and 8 are not showing the exact same slices of the tube but they have good correlation. The shortening is active in these sites, which means that it takes place before the major movements. A strong bipolar circumferential movement (twist) starts from both positions. Furthermore, the two first maximums of the radial displacements (narrowing) take place in these locations. All this evidence is in full agreement with the findings from the $26 \mathrm{hpf}$ dataset and highlights that the origin of the activation should be sought in these two places.

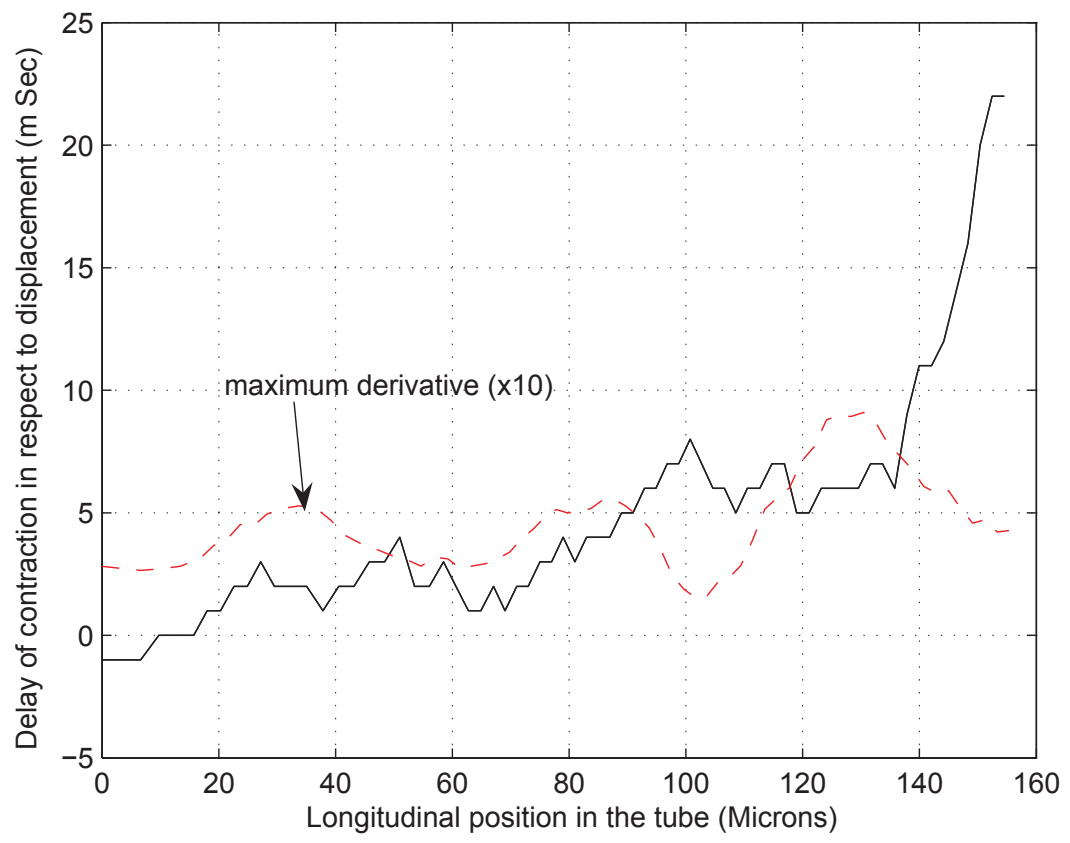

Figure 7. Delay between $90 \%$ of the maximum displacement for each cell and the time that myocardial cells start experiencing a $10 \%$ shortening strain or more (provided it is not less than one third of the maximum strain). Longer delays are known as a sign of passive behavior. Therefore only a small beginning portion of the tube (almost $20 \mu \mathrm{m}$ ) is active. However, the small delay near the $60 \mu \mathrm{m}$ position may show some active behavior there. The red dashed line represents the maximum derivative of the longitudinal displacement (or, equivalently, the shortening strain). It supports the existence of some activity near $60 \mu \mathrm{m}$. Its frequent alteration also may be related to some kind of spiral structure in the tube. It also may show different material properties in some points. 

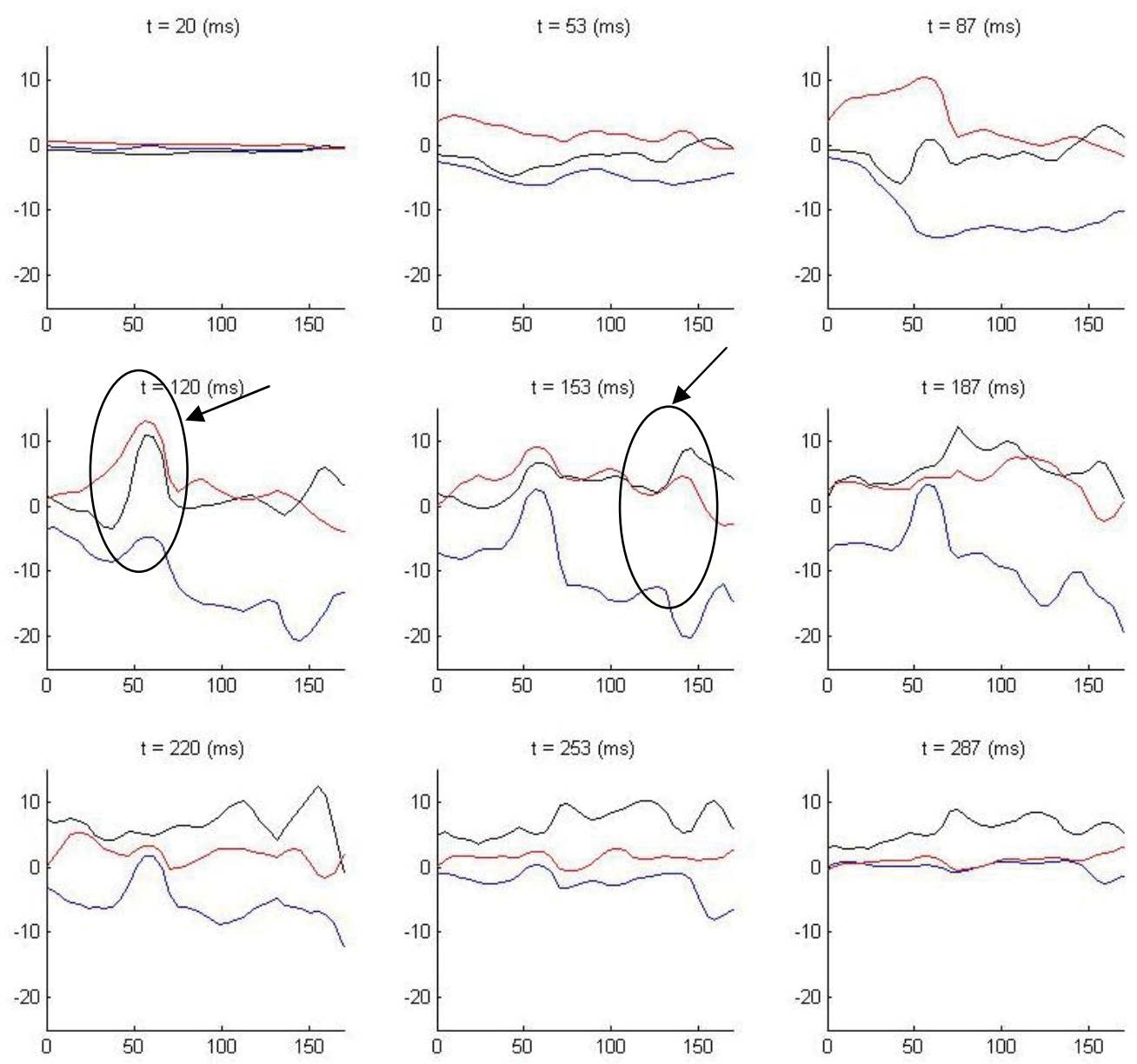

Figure 8. The longitudinal, radial, and circumferential displacements for the $34 \mathrm{hpf}$ dataset are shown in blue, red, and black, respectively. All units are in $\mu \mathrm{m}$. This figure emphasizes the findings from Figure 6.

\section{DISCUSSION}

Although the final goal for medical research is to understand the form-function relation of the adult heart, we have started from the embryonic heart not only because it seems easier to start with, but also because its fast changes will give us clues to understand the formation and structure of the heart. By showing several non uniformities in the function of the embryonic heart, we added evidences that the idea of a peristaltic pump cannot fully explain the behavior of the embryonic heart. Therefore, we need to find alternatives for that theory like the idea of the impedance pump [5].

By analyzing the above zebrafish embryonic data, we showed that, most likely, only the part at the very beginning of the tube (near the in-flow), almost the first 20 microns, and another place are active. The second one most probably is related to the separation of the atrium and ventricle. Rather these locations the rest of the tube seems mostly passive. This supports the idea of the impedance pump for the early stages of the embryonic heart.

Furthermore it shows that the time delay between displacement and shortening increases as the wave travels to the outflow site. However, the delay doesn't grow regularly. At a position of the tube about 60 microns from the beginning, the maximum contraction drops as well as the aforementioned delay. It may show an activity at that position, too. It is quite interesting to find out if there is any relation between this phenomenon and the fact that we expect the separation of the atrium and ventricle around that position. We also see another local minimum for the contraction in a site with a considerable delay (around $100 \mu \mathrm{m}$ ). It may be caused by an active element with different kind of material. Frequent alteration of the dashed line also may be related to kind of spiral structure in the tube. These are some of the open questions which need more research. 


\section{CONCLUSION}

Using computational methods we have provided suitable tools to investigate the structure-function interrelations on embryonic heart models. We used these methods, as well as strain-displacement analysis, on confocal laser scanning microscopy images of fluorescently labeled zebrafish hearts at two developmental stages. We have shown evidence for the hypothesis of a partially active, helical muscle structure, embedded in the heart tube. Hypothetical structures are suggested and tested based on the measured functionality at two different stages of embryonic heart growth.

Analyzing the images suggests that at $26 \mathrm{hpf}$, only $15 \%$ of the embryonic heart tube, in the caudal region (near the inflow) and probably another location around either 60 or 120 microns from the beginning of the tube are active and the rest appears to be mostly passive. Wave-like propagation of all three components of the displacement, especially in the circumferential direction, as well as almost-periodic changes of the maximum strain support the hypothesis of helical muscle structure embedded in the tube. Changes of geometry in the embryonic heart after several hours are used to verify speculations about the structure based on the earlier images and aforementioned methods.

\section{REFERENCES}

1. Buckberg G.D. et al., Left Ventricular Form and Function: Scientific Priorities and Strategic Planning for Development of New Views of Disease. Circulation, 110:333-336, (2004).

2. Steering, Handbook of physiology, section 2: Cardiovascular Systems.

3. Fishman, M. C., \& Chien, K. R. Development. 124, 2099-2117, (1997).

4. Gilbert, S. F. Developmental Biology., Sinauer Associates, Inc., Publishers, Sunderland, Massachusetts, 2000.

5. Hickerson, A.I. et al. Experiments in Fluids, in press.

6. Huang, C. J. et al. Developmental Dynamics. 228, 30-40, (2003).

7. M. Liebling, et al. J. Biomed. Optics, vol. 10, no. 5, eid 054001, 10 pages, (2005).

8. Amini A.A., Duncan J.S., Differential geometry for characterizing 3D shape change. SPIE Math Methods Med Imaging, 1768:170-181, (1992). 